\title{
Pod-Mounted Omnidirectional Traveling-Wave Antenna with High Conformability and Multioctave Bandwidth
}

\author{
Johnson J. H. Wang* and David J. Triplett \\ Wang Electro-Opto Corporation (WEO) \\ Marietta, Georgia 30067 USA \\ Phone: (770)-955-9311 Email: jjhwang@weo.com
}

\begin{abstract}
I. Introduction
Ultra-wideband antennas are increasingly more needed as wireless applications proliferate. These applications often require the antenna to be conformally mounted on a platform. Although many "frequency-independent" (FI) antennas were invented around 1950-1970, these FI antennas were not conformable to the surface of a platform. Mounting an FI antenna on an electrically conducting platform always resulted in major disruptions in its impedance and pattern.

The invention of the SMM (Spiral-Mode Microstrip) antenna in 1989 [1, 2] and the ensuing research efforts led by the senior author [3,4], as well as by some at several other major research institutions worldwide, have led to planar FI antennas conformal to planar platforms. For the planar FI antenna, the concept of self-complementary (SC) antenna is considered to be a more fundamental basis than other theories [5, 6]. However, the SC theory covers only the impedance of the antenna. To cover both impedance and radiation pattern, the concept of traveling-wave (TW) antenna was introduced $[3,4,6]$.
\end{abstract}

However, the TW antennas investigated so far have been of the planar type, or slightly curved planar antenna with a large radius of curvature. This paper presents a new antenna with high conformability; in other words, with a highly curved non-planar form factor (having a small radius of curvature). This new approach is attractive for applications in which the antenna must be mounted conformally on a small and highly curved platform, such as an instrument pod of an aircraft or a cell phone, and have multioctave bandwidth.

\section{Design Approach}

The new design approach, as shown in Fig. 1 by a photo of a scale model, is a TW antenna (without radome). It is mounted on, and strongly coupled to, a horizontal instrument pod (21.6-inch long and 5.1-inch in diameter) carried under a small airborne platform. The radiating aperture consists of an array of rectangular slots excited by a TW.

The antenna is designed to achieve an omnidirectional coverage in the azimuth plane (with the $z$ axis pointing to the zenith) with vertical polarization. For narrowband applications, a blade antenna or an annular slot can be used; the latter has advantages of conformability and lowprofile. None of them have multioctave bandwidth.

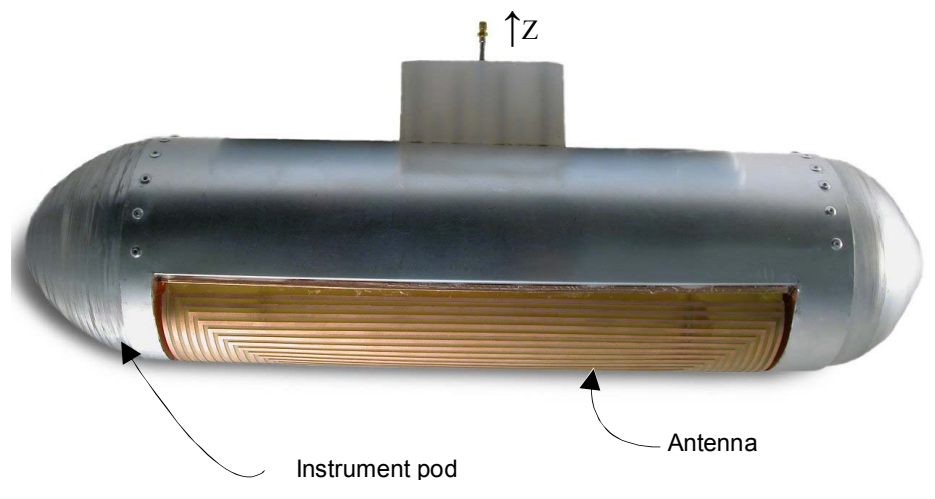

Fig. 1. Antenna on pod. 
The TW antenna is in essence an array of rectangular annular slots, which achieves multioctave bandwidth by shifting the radiation zone as the frequency changes. It is configured to have the TW continue onto the platform, thus extending the size of the antenna for enhanced gain pattern performance at low end of the operating frequencies.

\section{Measured performance over 0.44-4.40 GHz}

Although the research is still at an early stage, measured data on scale models obtained so far clearly demonstrated that this is a promising approach for a bandwidth of 10:1. Fig. 2 shows measured SWR data over 0.44-4.40 GHz. (In fact, similar impedance bandwidth up to 20:1 has been observed.)

Figs. 3 and 4 display exemplifying elevation patterns over 0.44-4.40 $\mathrm{GHz}$, measured at azimuth angles $\varphi$ $=0^{\circ}$ and $\varphi=90^{\circ}$, respectively. The polarization is predominantly vertical. The elevation patterns are generally similar to those of conical monopole and annular slot.

Fig. 5 shows exemplifying azimuth patterns over 0.44-4.40 GHz, measured at the horizon (elevation angle $\theta=90^{\circ}$ ). Ripples in azimuth patterns arise with increasing frequency, due to the elongated shape of the antenna.

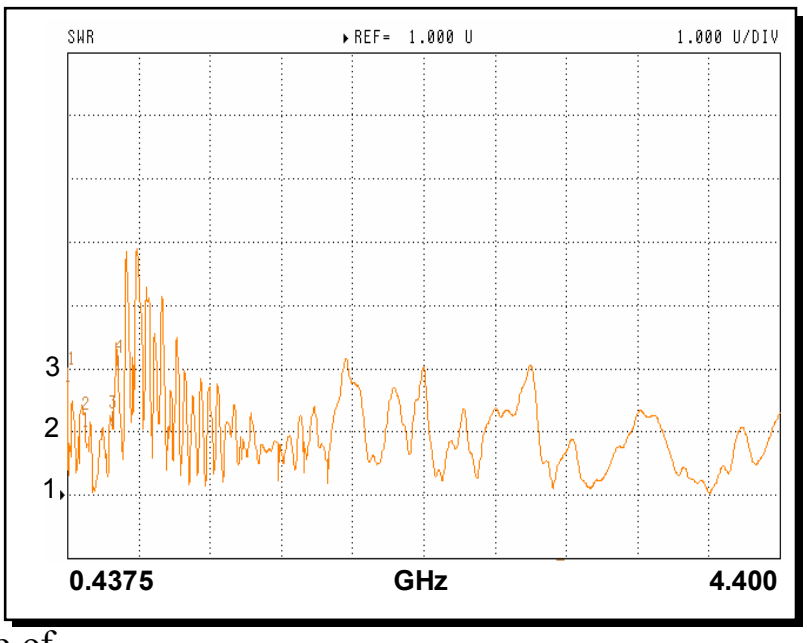

Fig. 2. Measured SWR over 0.44-4.40 GHz.

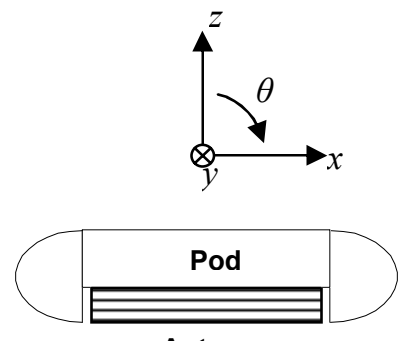

Antenna

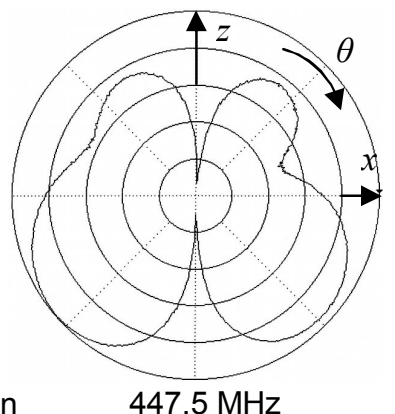

$5 \mathrm{~dB} /$ division
$447.5 \mathrm{MHz}$

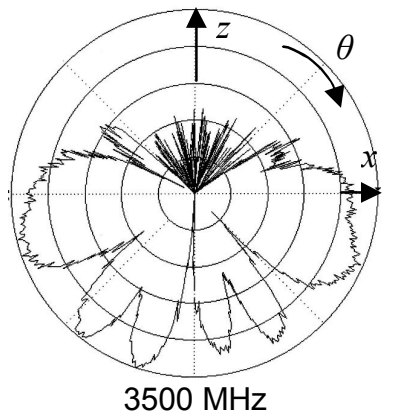

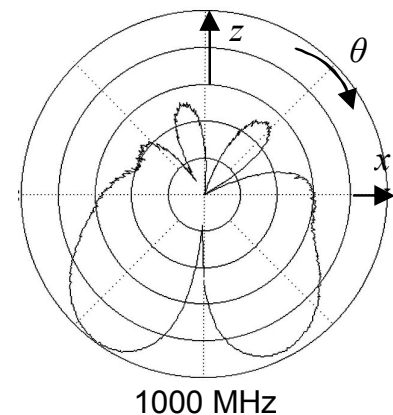

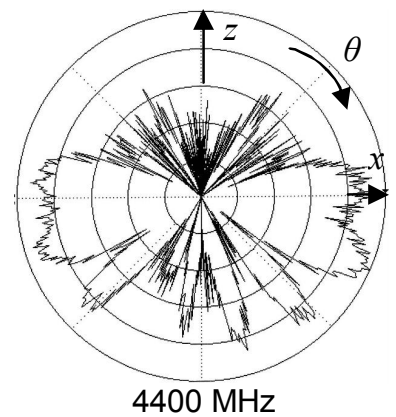

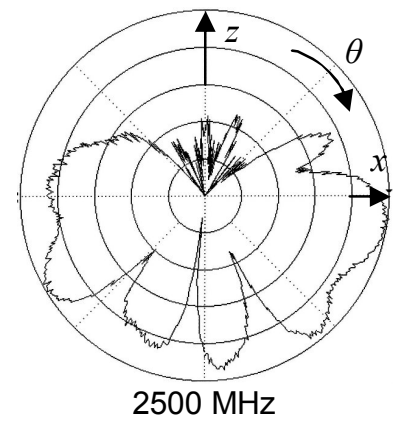

Figure 3. Typical measured elevation radiation patterns at $\varphi=0^{\circ}$ over $0.44-4.40 \mathrm{GHz}$. 

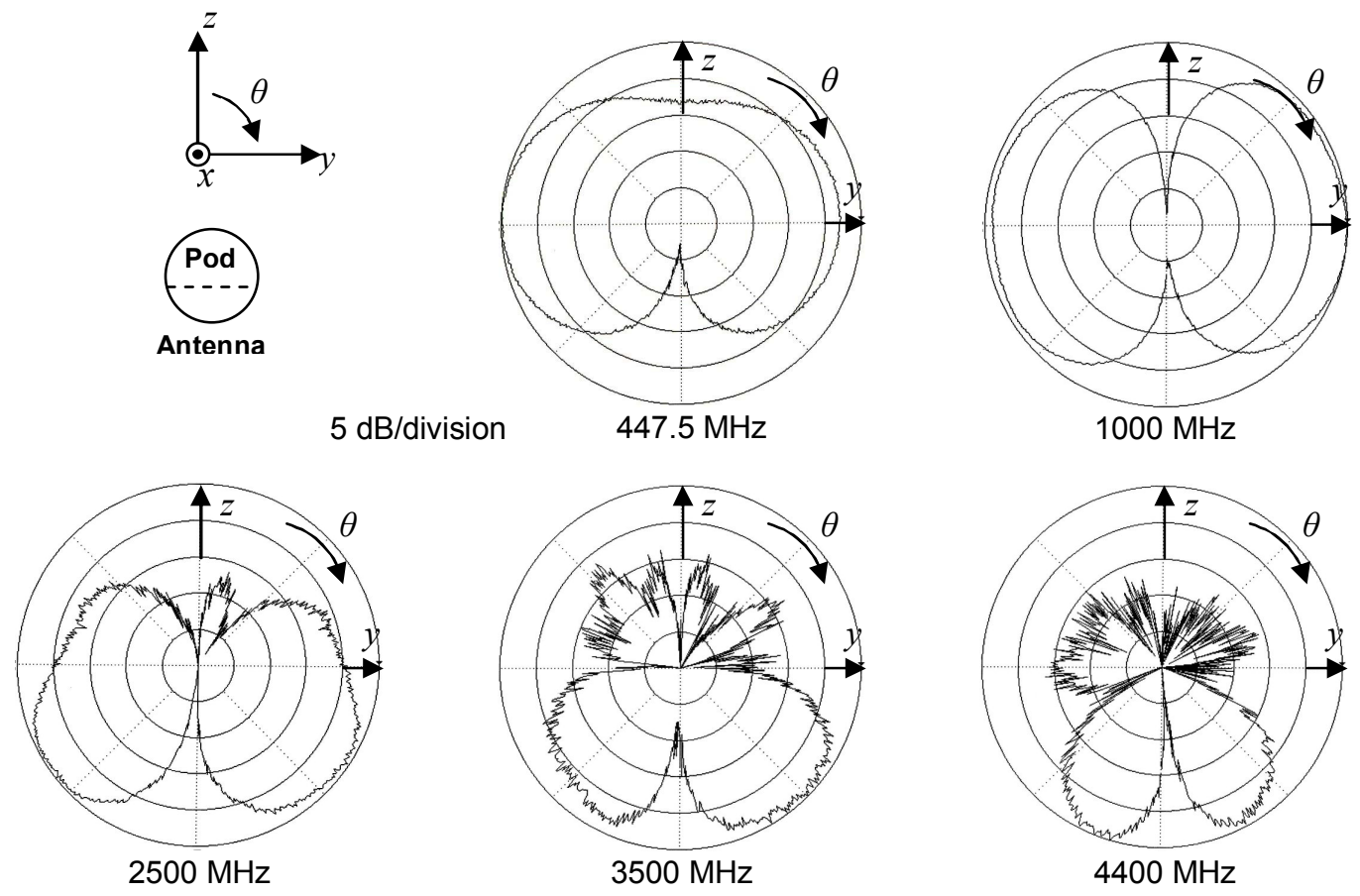

Fig. 4. Typical measured elevation radiation patterns at $\varphi=90^{\circ}$ over $0.44-4.40 \mathrm{GHz}$.
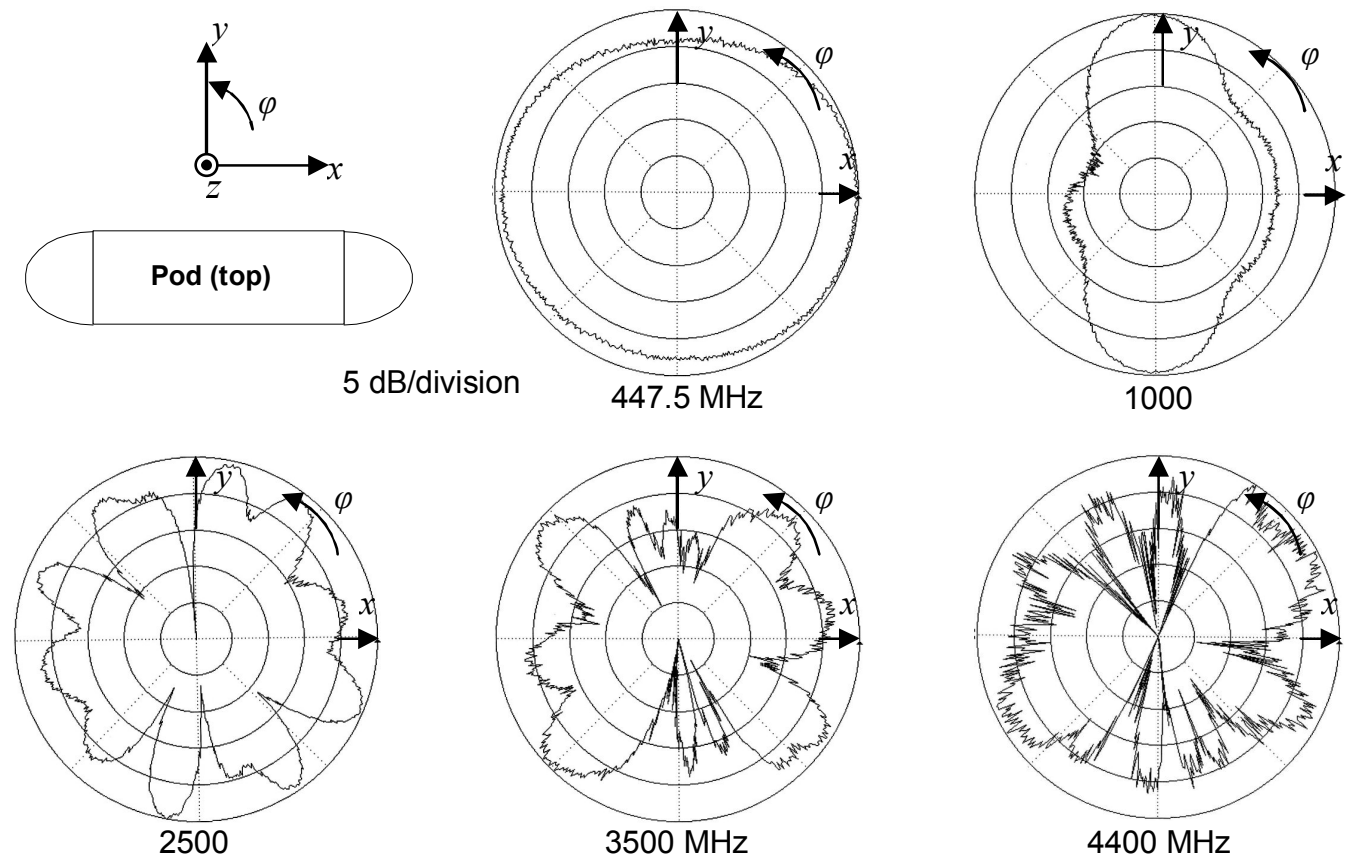

Fig. 5. Typical measured azimuth radiation patterns at $\theta=90^{\circ}$ over $0.44-4.40 \mathrm{GHz}$. 
Measured peak antenna gain at sampled frequencies appears to be generally consistent with the radiation pattern and impedance matching performance, generally around $2 \mathrm{dBiL}$ (or $2 \mathrm{dBi}$ since the polarization is predominantly vertical), dropping at the low end of frequencies due to the fundamental Chu limitation of electrically small antennas [7]. The current effort to enhance antenna gain at low end of the frequency by circumventing the Chu limitation will be discussed in a separate paper.

Noting the poor symmetry in the measured data for a symmetrical model, we believe the performance of this design can be considerably improved with more accurate fabrication. And impedance matching, for both the interior antenna feed and the exterior coupling with the pod, can be further improved to under 2:1 over the 10:1 bandwidth, and under 1.5:1 over selected operating bands.

\section{Conclusions}

A new design approach for a pod-mounted omnidirectional antenna was developed. It is a traveling-wave antenna with high conformability, strongly coupled with the instrument pod to enable it to circumvent the Chu limitation on the gain bandwidth due to antenna size. Scale model experiments exhibited impedance bandwidth up to 20:1 and gain pattern bandwidth of 10:1. Research is continuing, and considerable improvement in performance can be expected since the development is in its early stage and several potential improvements are obvious and straightforward.

\section{References}

1. J. J. H. Wang and V. K. Tripp, "Design of Multioctave Spiral-Mode Microstrip Antennas," IEEE Trans. Ant. Prop., March 1991.

2. J. J. H. Wang and V. K. Tripp, "Multioctave Microstrip Antenna," U.S. Patent No. 5,313,216, May 17, 1994.

3. J. J. H. Wang, "The Spiral as a Traveling Wave Structure for Broadband Antenna Applications," Electromagnetics, 20-40, July-August 2000; also U.S. Patents \#5,508,710, April 16, 1996, and \#5,621,422, April 15, 1997.

4. J. J. H. Wang, D. J. Triplett, and C. J. Stevens, "Broadband/Multiband Conformal Circular Beam-Steering Array," IEEE Trans. on Antennas and Prop., Vol. 54, No. 11, pp. 3338-3346, November 2006.

5. Y. Mushiake, "A Report on Japanese Development of Antennas: From the Yagi-Uda Antenna to Self-complementary Antennas," IEEE Antennas and Prop. Magazine, Vol. 46, August 2004; also in 2003 IEEE Antennas and Prop. Symp. Dig, June 2003.

6. J. J. H. Wang, "The Physical Foundation, Developmental History, and Ultrawideband Performance of SMM (Spiral-Mode Microstrip) Antennas," 2005 IEEE Antennas and Prop. Symp., Washington, DC, July 2005.

7. L. J. Chu, "Physical Limitations of Omnidirectional Antennas," J. Appl. Phys., Vol. 19, 1948. 